\title{
Adjustable Plasmonic Bandgap in One-Dimensional Nanograting Based on Localized and Propagating Surface Plasmons
}

\author{
Foozieh Sohrabi and Seyedeh Mehri Hamidi* \\ Magneto-plasmonic Lab, Laser and Plasma Research Institute, Shahid Beheshti University, \\ Daneshju Boulevard, Tehran
}

Corresponding Author's Email: m_hamidi@sbu.ac.ir

Received: Apr. 30, 2018, Revised: Apr. 13, 2019, Accepted: Jun. 29, 2019, Available Online: Dec. 27, 2019

DOI: 10.29252/ijop.13.2.97

\begin{abstract}
Compared to the long history of plasmonic gratings, there are only a few studies regarding the bandgap in the propagation of plasmonic surface waves. Considering the previous studies on interpretation of plasmonic bandgap formation, we discuss this phenomenon using the effect of both surface plasmon polariton (SPP) and localized surface plasmon (LSP) for our fabricated onedimensional metallic-polymeric grating. This structure is composed of metallic grating on the surface of PDMS with different concentration of embedded gold nanoparticles. By sweeping the incident angles, we have seen that the SPP, LSP and their coupling cause two gaps in reflection regime which are originated from SPP supported by thin film gold film and LSP supported by gold nanoparticles. The first gap is attributed to the patterned metallic film because it vanishes by increasing the nanoparticles which may destroy the pattern while the second gap can be formed by embedded nanoparticles because it becomes more considerable by raising the incubation time. Therefore, the drowning time of patterned samples (e.g. 24h, 48h, and 72h) in HAuCl4 plays the key role in adjustability of plasmonic bandgap. Notably, the interaction between SPP and LSP can be the origin of the shift in gap center from 300 to 550 . To best of over knowledge, this study is the first study on the plasmonic band gap as a function of both SPP and LSP.
\end{abstract}

KEYWORDS: One-dimensional grating, localized surface plasmon, propagative surface palsmon, gold nanoparticles.

\section{I.INTRODUCTION}

Although the history of the interaction between the light and metallic nanostructures dates back to more than a century [1-2], the concept of plasmonic band gap called the attention recently. The first physical interpretation of the existence of plasmonic band gap is stated by Barnes et al. [3] in 1996. In their paper, they stated that plasmonic bandgap is observed in a periodically modulated metal/dielectric interface when the Bragg vector (equal to $2 \pi /$ period) is twice the wave vector of SPP (kSPP) andthe forward and backward surface waves (i.e. surface plasmon polariton) form standing waves with field extrema located in the grating peaks and troughs $[3,4]$. These standing waves have two solutions with different energies creating a bandgap in the propagation of these surface modes. These plasmonic band gaps are located at the center and at the boundaries of the first Brillouin zone $\left(\mathrm{k}_{\mathrm{x}}=0\right.$ and $\left.\mathrm{k}_{\mathrm{x}}= \pm \frac{\pi}{\mathrm{d}}\right)$ [5]. As mentioned by Ref. [6], this is the higher harmonic components that plays the key factor for the formation of the plasmonic band gap. This necessity for higher harmonics can be understood if we consider a surface profile like

$$
\begin{aligned}
A(x)= & a_{1} \sin \left(k_{g} x+\phi_{1}\right)+a_{1} \sin \left(2 k_{g} x+\phi_{2}\right)+ \\
& a_{N} \sin \left(N k_{g} x+\phi_{N}\right)
\end{aligned}
$$


where $a_{N}$ is the amplitudes of corresponding harmonic components, $\phi_{N}$ is the relative phases and Bragg wave vectors $k_{g}=2 \pi /$ Period . Due to fabrication limitations, the emergence of these higher order harmonics are common. In fact the first two sentences play a key role: the $k_{g}$ component provides photon-plasmon coupling while $2 k_{g}$ component yields the gap.

Javaid and Iqbal [7] in their paper have interpreted the plasmonic bandgap by dividing the energy region in dispersion relation curve. They mentioned that at lower energies, SPP has dominantly the surface polaritonic nature but at higher energies, closer to $\hbar \omega s p$, it separates from light line and electromagnetic incident field is going to couple to coherent charge oscillations at the surface. At the energy of $\omega s p$, SPP is no longer propagating wave and it has almost the behavior of pure charge oscillation (i.e. surface plasmonic nature). This is above this energy that plasmonic band gap is observed where there are only imaginary solutions to the Maxwell's equations and we have radiation into the metal at Brewster's angle due to the positive sign of $\operatorname{Re}(\varepsilon)$ in this region.

Another interpretation for the emergence of plasmonic band gap is proposed by Ceglia et al. [8] in 2011.They stated that in an array of nanoslits there are three dominant modes: 1) Fabry-Perot cavity modes where most of the field localization is inside the slits 2) leaky surface waves at the surface of the grating and 3 ) hybrid modes created by the coupling of the first two modes (i.e. Fabry-Perot cavity resonances and surface waves). This hybridization is originated from the interference of the Fabry-Perot resonances with the Wood's anomaly (first-order diffracted wave propagating along the surface) that happens by choosing the pitch size equal to the Fabry-Perot resonance wavelength that provides. This mode hybridization can be the origin of observing two contradicted phenomena: extraordinary transmission and plasmonic bandgap. In its plasmonic band gap regime, light is impeded from entering into the slits and it is simultaneously coupled and back-reflected at the surface plasmon band gap. In other words, coupling to the cavity modes is reinforced due to the strong field localization at high energy plasmonic band edge while transmission is suppressed at low energy band edge due to weak coupling [8]. In addition to investigation of this mode analysis, Ceglia et al. [8] investigated the transmission of the grating with respect to the incident angle, aperture size of the slits and the grating thickness.

After the idea of investigating the coupling of cavity modes and surface plasmon resonances, Karademir et al. investigated the plasmonic band gap engineering of plasmon-exciton coupling[9]. Using the concept of plasmonic bandgap, they studied the resonant coupling between the molecular resonance of a Jaggregate dye and the plasmonic resonance of a textured metal film. They concluded that one-dimensional grating does not show a band gap except for the grating direction ( $\Gamma$ direction). Also, the coupling occurs when the molecular resonance energy level is outside of the bandgap along $\Gamma$ direction while inside it, this coupling is vanished. In brief, considering this fact that the propagation of surface modes is forbidden in plasmonic band gap and propagation is allowed with low group velocities and strong field localization at the band edge [8], different applications enter the sensing application. For instance, Grande et al. [10], investigated a novel biosensor based on the shift of the leaky surface plasmon mode at the edge of the plasmonic band gap raised from the analyte deposition on the metallic grating.

In this paper, we discuss on the combinatory effect of one-dimensional metallic pattern and localized surface plasmon of gold nanoparticles on the emergence of plasmonic bandgap. To best of over knowledge, this study is the first study on plasmonic band gap as a function of both SPP and LSP. 


\section{Fabrication Method}

In order to investigate the combinatory effect of SPP and LSP on plasmonic band gap, we used inexpensive and large-scale method of soft lithography to pattern one-dimensional plasmonic crystal [11]. Using this method, 0.6 gr poly (dimethylsiloxane) (PDMS) and 0.06 gr curing agent (Sylgard® 184 silicone elastomer kit) were weighted (10:1 ratio) and mixed. Then, the mixed PDMS/curing agent was added to our home-made template. For making this template, a one dimensional polycarbonate grating is extracted from a DVD-R (PRINCO, 4.7GB) with pitch of 741 $\mathrm{nm}$ and amplitude of $126 \mathrm{~nm}$ imaged by ARAAFM (0101/A) (not given here)[10]. This grating is used as the substrate and well-mixed PDMS/curing agent is added on top of it. Then we put the template in vacuum to remove the bubbles. Next step is heating at $500 \mathrm{C}-750 \mathrm{C}$ for about one hour. After 2 days, the cured PDMS with the area of $2 \times 2 \mathrm{~cm}^{2} 2 \times 2 \mathrm{~cm}^{2}$ is removed from the grating carefully by loosening the boundaries using blade. The AFM image of this patterned PDMS is seen in Fig. 1.
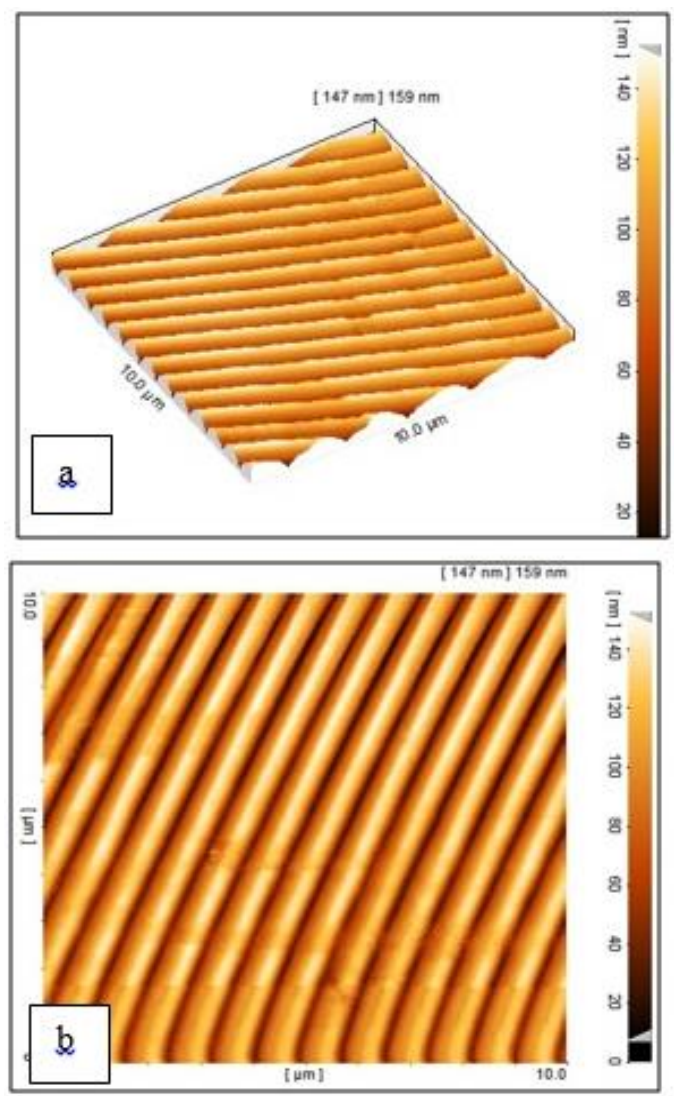

Fig. 1. The AFM image of 1D patterned PDMS fabricated using polycarbonate mold.

For synthesizing gold nanoparticles embedded in PDMS, we use in-situ synthesis proposed by Zhang et al. [13]. We divide this PDMS grating into 4 equal pieces using blade and then incubatedall pieces except one in the $\mathrm{HAuCl} 4$ aqueous solution at room temperature. We pull out these samples in $24 \mathrm{~h}$, $48 \mathrm{~h}$ and $72 \mathrm{~h}$. We have seen that the color of incubated PDMS samples turned to pale red and by increasing the incubation time, they become violet red. The chemical interpretation of this change is that the crosslinking of PDMS originates from reaction between the silicon hydride $(\mathrm{Si}-\mathrm{H})$ groups in the curing agent and vinyl groups ( $\mathrm{Si}-\mathrm{CHLCH} 2$ ) in the monomer[13] and these are the residual curing agent in the PDMS matrix ( $\mathrm{Si}-\mathrm{H}$ group) that reduces $\mathrm{HAuCl} 4$. The reaction is as follows:

$$
\begin{aligned}
& 3(\mathrm{Si}-\mathrm{H})+3 / 2 \mathrm{H}_{2} \mathrm{O}+2 \mathrm{AuCl} l_{4}^{-} \rightarrow \\
& 3 / 2-\mathrm{Si}-\mathrm{O}-\mathrm{Si}-2 \mathrm{Au}+8 \mathrm{Cl}^{-}+6 H^{+}
\end{aligned}
$$

The color change in PDMS means that the nanoparticles are synthesized in PDMS and PDMS allows AuCl4- anions to diffuse in it and reacted with the mentioned residuals. Darker Red color means that by raising incubation time, the number of nanoparticles enhances. However, if this time is very long, the possibility of aggregated nanoparticles increases.

The AFM images of the sample with incubation time of $48 \mathrm{~h}$ is given in Fig. 2. As it is clear, the formation of gold nanoparticles in upper regions of PDMS can destroy the pattern to some extent. After providing onedimensional $\mathrm{Au}$ NP@PDMS grating composite, $30 \mathrm{~nm}$ gold is deposited on it using sputtering. Our fabrication method benefits from this fact that it does not need any additional reducing/stabilizing agents and moreover, all four samples (i.e. 24, 48, 72 AuNP@PDMS samples and patterned PDMS without NPs) are prepared from one PDMS film. In other words, these four samples are 
prepared by dividing one PDMS film into four parts.

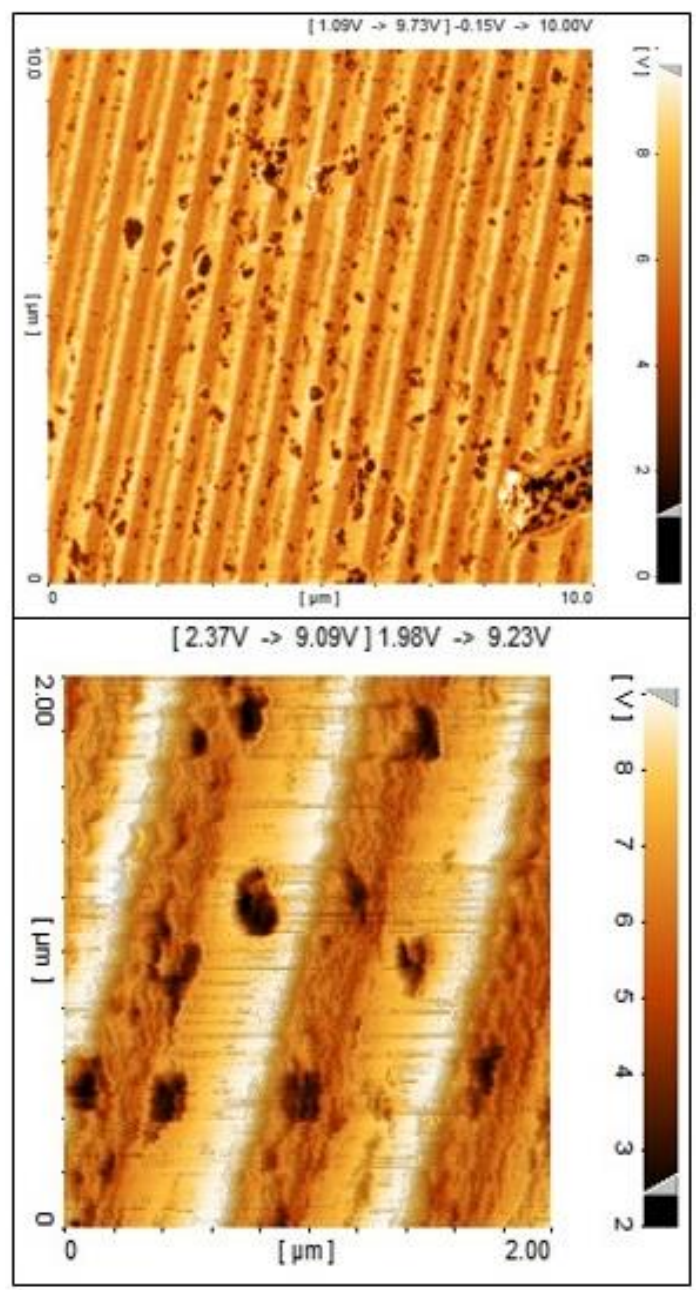

Fig. 2. The AFM image of $1 \mathrm{D}$ patterned PDMS with gold nanoparticles on its surface and inner part. The incubation time is $48 \mathrm{~h}$.

\section{III.RESULTS AND DISCUSSION}

In order to investigate the plasmonic bandgap, we use reflection spectrum with angular sweep between 200 and 700 under p-polarized, broadband incident wavelength. By selecting the wavelength interval of 550-650, the four samples have responses seen in Fig. 3. The suppression of reflection is clear in patterned PDMS without $\mathrm{Au}$ NPs and $72 \mathrm{~h}$ AuNP@PDMS samples at about 300 and 550, respectively. The below pattern of gold film helps to the coupling of incident light to the surface plasmons. The observed dip in Fig. 4 (a) can be interpreted as the emergence of forbidden region in reflection (plasmonic band gap) originated from thin patterned gold film.
When we consider 24 and 48 AuNP@PDMS samples where gold nanoparticles are located inside or at the upper surface of the pattern, we do not observe significant bandgap. However, 72h AuNP@PDMS sample with larger number of nanoparticles provides a sharp reflection dip at 550 which cannot be explained by the dip previously observed at 300 for patterned PDMS without Au NPs. We may say that the presence of nanoparticles helps to have palsmonic bandgap at the range of 450600 when we go from $24 \mathrm{~h}$ to $72 \mathrm{~h}$. Roughly, we can say that we do not have plasmonic bandgap in the mentioned range (450-600) for $24 \mathrm{~h}$ where the concentration of Au NPs is low.

It is clear that in pure patterned sample with 30 $\mathrm{nm} \mathrm{Au}$ film without the presence of Au NPs, we do not have well-defined plasmonic bandgap in the range of $450-600$ which is similar to the sample of $24 \mathrm{~h}$ with low concentration of NPs (in contrast to samples of $48 \mathrm{~h}$ and $72 \mathrm{~h}$ ). However, we have sharp dip in 300 in pure patterned sample with $30 \mathrm{~nm} \mathrm{Au}$ coat which is seen in $24 \mathrm{~h}$ sample but it is not as remarkable as this sample. The fact is that this sharp dip is occurred to the presence of thin Au film which is destroyed to some extent in samples with NPs due to the aggregation of NPS on some parts of the pattern. The SPP excitation is occurred due to the pattern of $\mathrm{Au}$ film. Therefore, the destruction of pattern does interfere the SPP excitation and makes the sharp dip less significant. Notably, LSP-SPP coupling can be the origin of the shift in gap center from 300 to 550 .

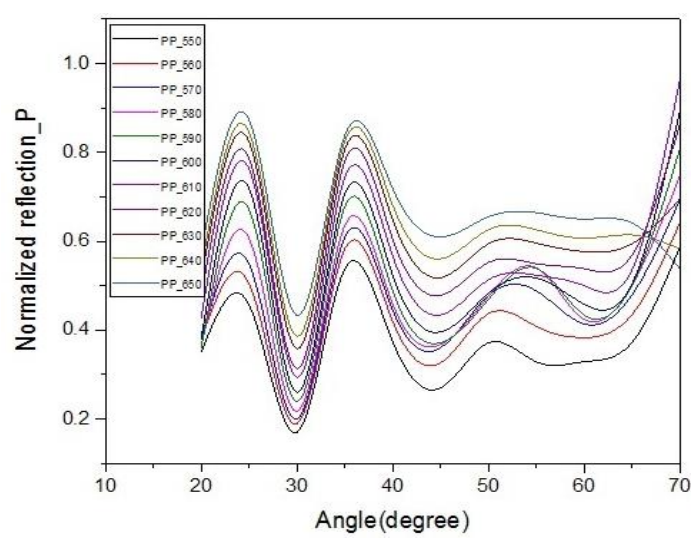

(a) 


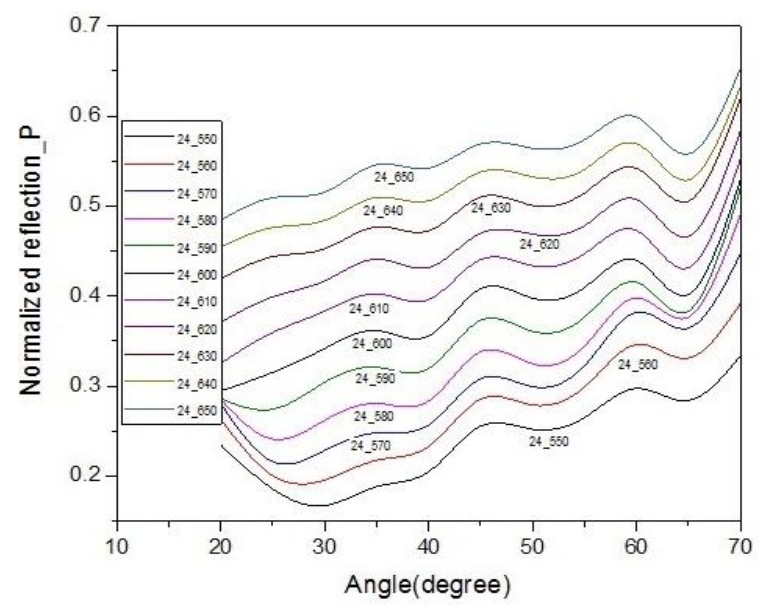

(b)

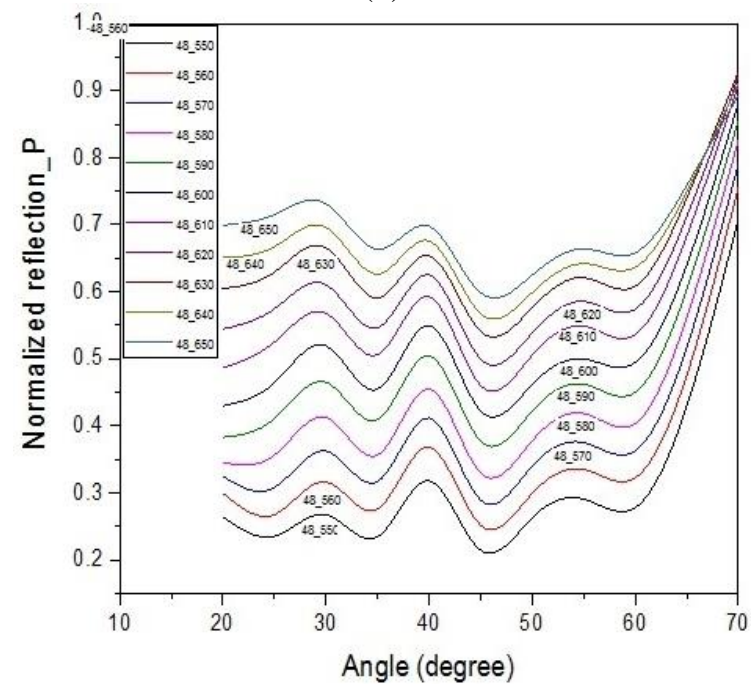

(c)

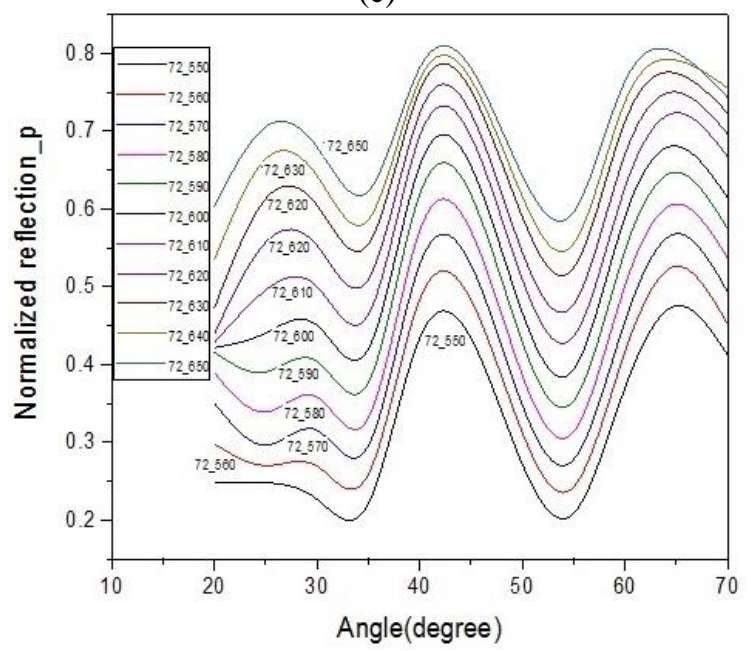

(d)

Fig. 3. Normalized reflection with respect to the incident angle for four samples: patterned PDMS without Au NPs (a) 24h AuNP@PDMS (b) 48h AuNP@PDMS (c) 72h AuNP@PDMS (d). The hour refers to the incubation time.

\section{IV.CONCLUSION}

In this study, the plasmonic bandgap is investigated for our fabricated 1D metallicpolymeric grating with embedded gold nanoparticles supporting both SPP and LSP. By angular sweep of reflection spectrum with broadband light source, two considerable gaps are observed with the gap center at 300 and 550. The origin of one of these gaps is attributed to the SPP of metallic gold film and the origin of the second gap can be the LSP supported by gold nanoparticles embedded in patterned PDMS and LSP-SPP coupling. The gap origins can be interpreted by adjusting the incubation time of PDMS samples in $\mathrm{HAuCl} 4$ solution (i.e. $24 \mathrm{~h}, 48 \mathrm{~h}$ and $72 \mathrm{~h}$ ). The increase of incubation time means the augmentation in the amount of nanoparticles formed in the PDMS which can subsequently destroy the pattern. Pattern destruction can affect the SPP because it is excited using this pattern and there is no prism coupling. For purely patterned sample without nanoparticles, the gap is centered at 300 while for $72 \mathrm{~h}$ with large number of isolated and aggregated nanoparticles, it is located at 550. LSP-SPP coupling may be the origin of the shift in gap center from 300 to 550 . In this paper, we try to show the combinatory effect of SPP and LSP on emergence of plasmonic bandgap in an innovative fashion.

\section{References}

[1] S.M. Hamidi, M. Zamani, and H. Goudarzi, "One-step construction and examination of one-dimensional plasmonic chromium nanograting," Opt. Commun. Vol. 345, pp. 47-51, 2015.

[2] X. Yang and D. Liu, "Sensitivity enhancement of surface plasmon resonance sensors through planar metallic film closely coupled to nanogratings," Chin. Opt. Lett. Vol. 5, pp. 563-565, 2007.

[3] W.L. Barnes, T.W. Preist, S.C. Kitson, and J.R. Sambles, "Physical origin of photonic energy gaps in the propagation of surface plasmons on gratings," Phys. Rev. B, Vol. 54, pp. 6227-6244, 1996. 
[4] S.C. Kitson, W.L. Barnes, and J.R. Sambles, "Full Photonic Band Gap for Surface Modes in the Visible," Phys. Rev. Lett. Vol. 77, pp. 2670-2673, 1996.

[5] C. Billaudeau, S. Collin, F. Pardo, N. Bardou, and J.L. Pelouard, "Tailoring radiative and non-radiative losses of thin nanostructured plasmonic waveguides," Opt. Express, Vol. 17, pp. 3490-3499, 2009.

[6] T. Iqbal and S. Afsheen, "Plasmonic Band Gap: Role of the Slit Width in 1D Metallic Grating on Higher Refractive Index Substrate," Plasmonics," Vol. 11, pp. 885 893, 2016.

[7] M. Javid and T. Iqbal, "Plasmonic Bandgap in 1D Metallic Nanostructured Devices," Plasmonics, Vol. 11, pp. 167-173, 2016.

[8] D.d. Ceglia, M.A. Vincenti, M. Scalora, N. Akozbek, and M.J. Bloemer, "Plasmonic band edge effects on the transmission properties of metal gratings," AIP Advances, Vol. 1, pp. 032151 (1-16), 2011

[9] E. Karademir, S. Balci, C. Kocabas, and A. Aydinli, "Plasmonic band gap engineering of plasmon; exciton coupling," Opt. Lett. Vol. 39, pp. 5697-5700, 2014.

[10] M. Grande, M.A. Vincenti, T. Stomeo, G. Morea, R. Marani, V. Marrocco, V. Petruzzelli, A. D’Orazio, R. Cingolani, M.D. Vittorio, D.d. Ceglia, and M. Scalora, "Experimental demonstration of a novel bio-sensing platform via plasmonic band gap formation in gold nano-patch arrays," Opt. Express, Vol. 19, pp. 21385-21395, 2011.

[11]F. Sohrabi and S.M. Hamidi, "Fabrication methods of plasmonic and magnetoplasmonic crystals: a review," Europ. Phys. J. Plus, Vol. 132, pp. 1-21, 2017.

[12]S.M. Hamidi and M. Zamani, "Use of nanostructures based on silver nanospike prepared by oblique angle deposition as broadband optical polarizer," Optical Engineering, "Vol. 54, p. 107104 (1-6), 2015.
[13] Q. Zhang, J.J. Xu, Y. Liu, and H.Y. Chen, "Insitu synthesis of poly (dimethylsiloxane)-gold nanoparticles composite films and its application in microfluidic systems," Lab Chip, Vol. 8, pp. 352-357, 2008.

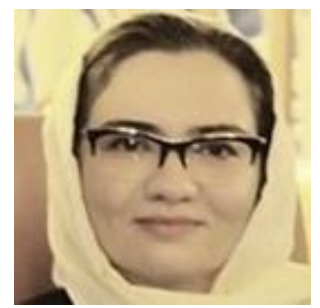

Foozieh Sohrabi has obtained her BS in physics and MS in photonics engineering at University of Tabriz in 2011 and 2013, respectively. Subsequently, she was awarded her $\mathrm{PhD}$ degree in photonics from Shahid Beheshti University. Her research interest is focused on optical sensing, plasmonics, neurophotonics and microfluidics.

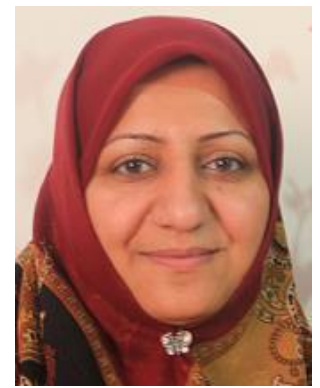

Seyedeh Mehri Hamidi received her Ph.D. degree in photonics from Laser and Plasma Research institute, Shahid Beheshti University, Tehran, Iran, in 2009. She is currently the director of magneto-plasmonic Lab. at Laser and Plasma Research Institute. She has worked on the research fields of magneto-plasmonic, nanophotonics, neurophotonics, piezotonics, photonic and magnetophotonic crystals, Surface Plasmon Resonance, dielectric and magnetic nanostructures. 